\title{
Avaliação da função pancreática em pacientes com diabetes melito tipo 1 de acordo com a duração da doença
}

\author{
Pancreatic function assessment in type 1 diabetes \\ mellitus patients according to disease duration
}

Joana R. Dantas ${ }^{1,2}$, Mirella H. Almeida ${ }^{2}$, Bianca Barone ${ }^{1,2}$, Felipe Campos ${ }^{2}$, Rosane Kupfer ${ }^{1}$, Adolpho Milech², Lenita Zajdenverg², Melanie Rodacki ${ }^{1,2}$, José Egídio P. de Oliveira ${ }^{2}$

1 Instituto Estadual de Diabetes e Endocrinologia Luiz Capriglione (IEDE) 2 Serviço de Nutrologia, Hospital Universitário Clementino Fraga Filho (HUCFF), Universidade Federal do Rio de Janeiro (UFRJ); Rio de Janeiro, RJ, Brasil

\section{Correspondência para: Joana R. Dantas Rua Engenheiro Cortes Sigaud, 198, apto. 102 - Leblon 22450-150 Rio de Janeiro, RJ joanardantasp@ig.com.br}

Recebido em 13/Mai/2008 Aceito em 13/Nov/2008

\section{RESUMO}

Os pacientes com diabetes melito tipo 1 (DM1) podem apresentar secreção residual de insulina por longos períodos, o que tem sido associado a prognóstico mais favorável. Objetivo: Avaliar a secreção de insulina por meio da dosagem de peptídeo C (PC) em pacientes com DM1 de curta ( $\leq 5$ anos; grupo 1) e longa (> 5 anos; grupo 2) duração da doença. Pacientes e Métodos: Voluntários com DM1 coletaram sangue em jejum e 6 minutos após a infusão de glucagon para dosagem de PC, HbA1c e anti-GAD. Resultados: Foram avaliados 43 pacientes, 22 no grupo 1 e 21 no grupo 2. Secreção de insulina preservada ( $P C>1,5 \mathrm{ng} / \mathrm{mL}$ ) foi identificada em seis $(13,9 \%)$ e oito $(18,6 \%)$ casos nas coletas basal (PC1) e após estímulo (PC2), sem diferença entre os grupos ( $p=0,18$ e 0,24). PC1 foi detectável $(>0,5 \mathrm{ng} / \mathrm{mL}$ ) em $13(30,2 \%)$ e PC2 em $18(41,9 \%)$ casos, mais frequentes no grupo 1 do que no 2 ( $p=0,045$ para PC $1 / p=0,001$ para PC2). Os títulos de PC1 $(1,4 \pm 0,8$ versus $1,2 \pm 1,0 ; p=0,69)$ ou PC2 $(1,8 \pm 1,5$ versus $1,7 \pm 0,8 ; p=0,91)$ não diferiram entre os grupos. No grupo 1 houve correlação inversa entre tempo de doença e PC2 ( $R=-0,58 ; p=0,025)$. Conclusão: Uma proporção significativa dos pacientes com DM1 apresenta secreção residual de insulina, especialmente nos primeiros cinco anos da doença. Tais indivíduos representam a população ideal para estudos visando à prevenção secundária da doença. Arq Bras Endocrinol Metab. 2009;53(1):64-71.

Descritores

Diabetes melito tipo 1; peptídeo C; secreção de insulina; tempo de doença

\section{ABSTRACT}

Patients with type 1 diabetes (T1D) may exhibit some residual insulin secretion for many years after their diagnosis. This has been associated with a more favorable prognosis. Objective: To analyze insulin secretion in individuals with T1D using C-peptide (CP) response to glucagon and comparing patients with recent onset ( $\leq 5$ years - Group 1) and long-standing disease ( $>5$ years - Group 2 ). Methods: Subjects with T1D had their blood sampled before (fasting) and 6 minutes after glucagon infusion for $\mathrm{CP}, \mathrm{HbA1c}$ and anti-GAD measurement. Results: Forty-three individuals were evaluated, 22 in Group 1 and 21 in Group 2. Preserved insulin secretion $(C P>1.5 \mathrm{ng} / \mathrm{mL}$ ) was observed in $6(13.9 \%)$ and in $8(18.6 \%)$ patients before (CP 1) and after (CP 2) glucagon stimulus, respectively, showing no difference between the groups ( $p=0.18$ and 0.24$)$. CP 1 and CP 2 were detectable $(>0.5$ $\mathrm{ng} / \mathrm{dL})$ in $13(30.2 \%)$ and $18(41.9 \%)$ patients, respectively. Both were more frequent in Group 1 than in Group 2 ( $p=0.45$ for $C P 1 / p=0.001$ for CP 2). Similar serum levels where seen between the groups, both before and after stimulus ( $1.4 \pm 0.8$ vs. $1.2 \pm 1.0 ; p=0.69$ and $1.8 \pm 1.5$ vs. $1.7 \pm 0.8 ; p=0.91)$. Group 1 presented an inverse correlation between disease duration and CP $2(R=-0.58 ; p=0.025)$. Conclusion: A significant number of patients with T1D have detectable residual insulin secretion, especially in the first 5 years of disease. These subjects are an ideal population for clinical trials that target the prevention of $\beta$ cell function loss in T1D. Arq Bras Endocrinol Metab. 2009;53(1):64-71.

Keywords

Type 1 diabetes; C-peptide; insulin secretion; disease duration 


\section{INTRODUÇÃO}

$\mathrm{O}$ diabetes melito tipo l (DMl) é uma doença crônica caracterizada pela destruição imunomediada progressiva das células $\beta$ das ilhotas pancreáticas, o que provoca secreção insuficiente de insulina. Os fatores imunogenéticos e ambientais associados ao início e à perpetuação da lesão pancreática associada ao $\mathrm{DMl}$ ainda não foram completamente esclarecidos $(1,2)$. A velocidade de progressão deste processo pode ser variável, evoluindo com a perda da capacidade de secreção insulínica em alguns meses ou até em vários anos. Além da destruição da massa de células $\beta$, pode haver comprometimento da função secretória das células remanescentes, de maneira transitória ou permanente $(3,4)$.

Uma das principais limitações dos estudos que avaliam a secreção de insulina em pacientes com DMl é a impossibilidade de mensurar a massa de células $\beta$ in vivo. Embora modelos animais venham sendo utilizados para elucidar questões acerca deste tema, nem sempre é possível extrapolar as informações obtidas para o DMl humano. Dessa forma, métodos indiretos para avaliação da massa de células $\beta$ e da função pancreática em humanos vêm sendo desenvolvidos e aprimorados. Não obstante a possibilidade de comprometimento funcional das células $\beta$ preservadas, a capacidade de secreção de insulina mostrou ter correlação aproximada com a massa de células $\beta$ utilizada no transplante de ilhotas nos pacientes com DMl (5).

A avaliação da função das células $\beta$ pode ser feita por diferentes métodos. O uso da dosagem da insulina no sangue periférico, com esta finalidade, tem limitações importantes: $40 \%$ a $60 \%$ sofrem efeito da primeira passagem pelo fígado, o seu clearance periférico varia de acordo com as condições metabólicas e não é possível diferenciar a insulina endógena da exógena ou da pró-insulina (6). O método mais adequado, aceito e clinicamente validado para a mensuração da função das células $\beta$ sob condições ideais é a dosagem do peptídeo C (PC) (7). Esta molécula é cossecretada na circulação portal em quantidade equimolar à da insulina, e não sofre metabolização hepática. Sua dosagem possui alta reprodutibilidade e variabilidade relativamente baixa (5-7), podendo ser basal ou sob estímulo, com glicose, refeição-padrão ou secretagogos de insulina, como o glucagon $(6,7)$. Os testes com estímulo apresentam boa correlação entre si e parecem ser superiores à avaliação basal (7). O teste com glucagon representa estímulo suprafisiológico, mas podem ter algumas vantagens sobre os demais, como maior rapidez, menor influência da glicotoxicidade, simples realização técnica e boa reprodutibilidade. Além disso, é teste seguro, que apresenta efeitos colaterais leves e transitórios (5-7).
Estudos recentes têm demonstrado função residual das células $\beta$ (por meio da dosagem de PC) em uma proporção significativa de indivíduos com $\mathrm{DMl}$, tanto ao diagnóstico quanto após vários anos da doença (5). A preservação desta capacidade de secreção seria atrativa para evitar a necessidade de insulinoterapia ou até curar o DM. Entretanto, mesmo sem atingir este objetivo, a manutenção de alguma função residual poderia ter vantagens importantes, como melhor controle metabólico, menor frequência de hipoglicemia e menor risco de complicações microvasculares (6-10). A maioria dos estudos acerca da função pancreática no DMl foi realizada em populações caucasianas ou de origem asiática. Ainda é pouco conhecido se estes resultados podem ser extrapolados para populações de outras etnias, como a população brasileira. Portanto, o objetivo deste estudo foi avaliar a capacidade de secreção pancreática em pacientes com DMl de população multiétnica, por meio da dosagem de PC basal e pós-estímulo com glucagon, comparando indivíduos com curta e longa duração de doença. Como também é desconhecido o papel dos autoanticorpos na preservação da função pancreática, pesquisou-se ainda a influência da positividade para o anti-GAD neste contexto. Este anticorpo foi escolhido porque, ao contrário dos demais autoanticorpos, pode permanecer positivo por tempo bastante prolongado (11) e parte da população estudada apresentava DMl por diversos anos.

\section{PACIENTES E MÉTODOS}

Foram estudados 43 voluntários com diagnóstico de DMl estabelecidos pelos critérios da American Diabetes Association (ADA) (12) em acompanhamento no Ambulatório de Diabetes do Instituto Estadual de Diabetes e Endocrinologia Luiz Capriglione (IEDE) e do Hospital Universitário Clementino Fraga Filho (HUCFF) da Universidade Federal do Rio de Janeiro (UFRJ). O protocolo foi aprovado pelo Comitê de Ética e Pesquisa de ambas as instituições.

Todos os pacientes assinaram o termo de consentimento livre e esclarecido. Os dados clínicos e epidemiológicos foram obtidos em questionários específicos e prontuário médico. Para etnia, os pacientes foram classificados como brancos e não-brancos, tendo em vista que a população brasileira é multiétnica, o que impossibilita diferenciação mais específica $(13,14)$.

Foram coletados $15 \mathrm{~mL}$ de sangue em jejum para dosagem de PC basal, hemoglobina glicada $(\mathrm{HbAlc})$, glicemia e anti-GAD. A seguir foi realizada infusão de $1 \mathrm{mg}$ de glucagon intravenoso, com nova coleta de $8 \mathrm{~mL}$ de sangue após 6 minutos para dosagem de PC pós-estímulo. Os in- 
divíduos com glicemia capilar $<70$ e $>200 \mathrm{mg} / \mathrm{dL}$ foram excluídos, para minimizar a interferência de hipo e hiperglicemias agudas nos resultados. A dosagem do PC foi feita por meio de quimiolumiescência/immunolite/DPC, com limite de detecção de $0,5 \mathrm{ng} / \mathrm{mL}$, coeficiente de variação inter e intraensaios de $7,6 \%$ e $8,2 \%$, respectivamente. A dosagem do anti-GAD foi realizada por radioimunoensaio, considerado positivo se $>1,0 \mathrm{U} / \mathrm{mL}$.

Segundo critérios da Immunology of Diabetes Society, foi considerada a secreção de insulina preservada se $\mathrm{PC} \geq$ $1,5 \mathrm{ng} / \mathrm{mL}(0,5 \mathrm{nmol} / \mathrm{l})$ e PC detectável se $\geq 0,5 \mathrm{ng} / \mathrm{mL}$ $(0,2 \mathrm{mmol} / \mathrm{L})$, incluindo-se os indivíduos com secreção de insulina preservada neste grupo.

Comparações entre estas variáveis foram estabelecidas entre pacientes com curta ( $\leq 5$ anos) e longa ( $>5$ anos) duração da doença (grupos 1 e 2, respectivamente). A análise descritiva foi feita com cálculo de medidas centrais e de dispersão da amostra (média \pm 1 desvio-padrão). Para comparação entre os grupos, foram utilizados os testes do qui-quadrado e de Mann-Whitney $U$ para variáveis categóricas e contínuas, respectivamente. As correlações foram calculadas por meio do coeficiente de Spearman. O coeficiente de significância estatística foi de $\mathrm{p}<0,05$ e testes bicaudados foram usados na análise dos dados.

\section{RESULTADOS}

\section{CARACTERÍSTICAS DOS PARTICIPANTES}

As características clínicas e epidemiológicas dos pacientes estudados estão descritas na Tabela 1. Não houve diferenças entre os grupos quanto ao nível de controle glicêmico ou etnia. Enquanto no grupo 1 houve predomínio do sexo masculino, no grupo 2 o oposto foi observado. Pacientes com curta duração da doença eram também mais jovens, mas apresentavam idade de diagnóstico mais elevada.

\section{DOSAGEM DE PEPTÍDEO C}

A secreção basal de $\mathrm{PC}(\mathrm{PCl})$ estava preservada em seis pacientes $(13,9 \%)$ e detectável em $13(30,2 \%)$ casos. Após estímulo com glucagon (PC2), foram encontrados secreção de insulina preservada em oito $(18,6 \%)$ pacientes e PC detectável em 18 (41,9\%) pacientes. Estes resultados estão indicados na Tabela 2. Sexo, idade de diagnóstico, positividade do anti-GAD, presença de CAD ao diagnóstico e etnia não influenciaram estes resultados. Observouse correlação inversa da HbAlc com o PC2 $(\mathrm{R}=-0,50$; $\mathrm{p}=0,04)$, porém não com $\mathrm{PCl}(\mathrm{R}=-0,26 ; \mathrm{p}=0,39)$, conforme demonstrado na Figura 1.

Tabela 1. Características clínicas e epidemiológicas dos pacientes estudados.

\begin{tabular}{|c|c|c|c|c|}
\hline & $\begin{array}{l}\text { Grupo1 } \\
\mathrm{n}=22\end{array}$ & $\begin{array}{c}\text { Grupo } 2 \\
n=21\end{array}$ & $\begin{array}{c}\text { Total } \\
n=43\end{array}$ & p valor \\
\hline \multicolumn{5}{|l|}{ Sexo } \\
\hline Feminino $\mathrm{n}(\%)$ & $8(36,4 \%)$ & $16(76,2 \%)$ & $24(55,8 \%)$ & $p=0,014$ \\
\hline Masculino n(\%) & $14(63,6 \%)$ & $5(23,8 \%)$ & $19(44,2 \%)$ & \\
\hline \multicolumn{5}{|l|}{ Etnia } \\
\hline Branco $n(\%)$ & $11(50 \%)$ & $12(57,1 \%)$ & $23(53,5 \%)$ & $p=0,76$ \\
\hline Não-branco n(\%) & $11(50 \%)$ & $9(42,9 \%)$ & $20(46,5 \%)$ & \\
\hline Média de idade ao diagnóstico (em anos) & $15,4 \pm 5,7$ & $10,6 \pm 6,9$ & $13,1 \pm 6,7$ & $p=0,002$ \\
\hline Tempo médio de doença (em anos) & $1,7 \pm 1,4$ & $13,6 \pm 8,7$ & $7,5 \pm 8,6$ & $p<0,001$ \\
\hline Média de idade atual (em anos) & $17,2 \pm 5,5$ & $23,8 \pm 7,2$ & $20,4 \pm 7,1$ & $p<0,001$ \\
\hline CAD ao diagnóstico n (\%) & $5(22,7 \%)$ & $9(42,9 \%)$ & $14(32,5 \%)$ & $p=0,17$ \\
\hline IMC $\geq 25$ kg/m² n (\%) & $3(13,6 \%)$ & $8(38,1 \%)$ & $11(25,6 \%)$ & $p=0,14$ \\
\hline HbA1c (média \pm DP) & $9,31 \pm 2,77$ & $8,87 \pm 2,09$ & $9,09 \pm 2,43$ & $p=0,792$ \\
\hline
\end{tabular}

$C A D$ = cetoacidose diabética; IMC = índice de massa corporal; grupo 1: pacientes com curta ( $\leq 5$ anos) duração da doença; grupo 2: pacientes com longa (> 5 anos) duração da doença.

\begin{tabular}{|c|c|c|c|c|c|c|c|}
\hline & \multicolumn{2}{|c|}{ Total } & \multicolumn{2}{|c|}{ Grupo 1} & \multicolumn{2}{|c|}{ Grupo 2} & \multirow{2}{*}{$\begin{array}{c}\text { p valor } \\
\text { Grupo } 1 \text { versus } \\
\text { Grupo } 2 \\
\end{array}$} \\
\hline & $\begin{array}{l}\text { Basal } \\
\text { (PC1) }\end{array}$ & $\begin{array}{l}\text { Pós-estímulo } \\
\text { (PC2) }\end{array}$ & Basal (PC1) & $\begin{array}{l}\text { Pós-estímulo } \\
\text { (PC2) }\end{array}$ & $\begin{array}{l}\text { Basal } \\
\text { (PC1) }\end{array}$ & $\begin{array}{l}\text { Pós-estímulo } \\
\text { (PC2) }\end{array}$ & \\
\hline PC detectável & $13(30,2 \%)$ & $18(41,9 \%)$ & $10(45,5 \%)$ & $15(68,2 \%)$ & $3(4,3 \%)$ & $3(14,3 \%)$ & $\begin{array}{l}P C 1-p=0,045 \\
P C 2-p=0,001\end{array}$ \\
\hline PC preservado & $6(13,9 \%)$ & $8(18,6 \%)$ & $5(22,7 \%)$ & $6(27,3 \%)$ & $1(4,8 \%)$ & $2(9,5 \%)$ & $\begin{array}{l}P C 1-p=0,18 \\
P C 2-p=0,24\end{array}$ \\
\hline
\end{tabular}

RC1 = peptídeo C basal; PC2 = peptídeo C pós-estímulo com glucagon; grupo 1 = pacientes com curta duração da doença ( $\leq 5$ anos); grupo 2 = pacientes com longa duração da doença ( $>5$ anos). 
$1 A$

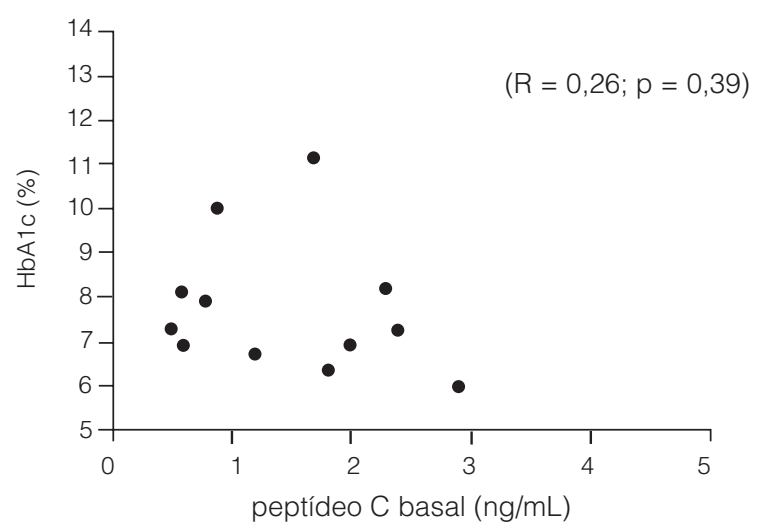

1B

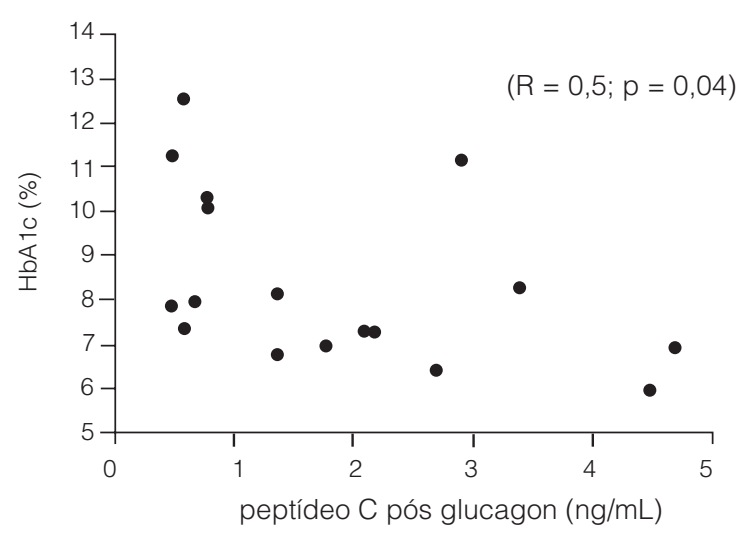

Figura 1. Correlação entre hemoglobina glicada ( $\mathrm{HbA} 1 \mathrm{c})$ e peptídeo $C(P C)$ basal (1A) ou pós-estímulo com glucagon $E V(1 B)$.

\section{COMPARAÇÃO DA FUNÇÃO PANCREÁTICA ENTRE PACIENTES COM CURTA E LONGA DURAÇÃO DE DOENÇA}

O PC detectável, basal ou após estímulo, foi mais frequente no grupo 1 (DMl de curta duração) do que no 2 (longa duração). Entretanto, a presença de secreção de insulina preservada basal ou pós-estímulo foi semelhante em ambos. Estes dados estão indicados na Tabela 2. Níveis similares de PC1 e PC2 também foram detectados entre os pacientes com diagnóstico de curta e longa duração $(1,4$ $\mathrm{ng} / \mathrm{mL} \pm 0,8$ versus $1,2 \mathrm{ng} / \mathrm{mL} \pm 1,0 ; \mathrm{p}=0,69$ e $1,8 \mathrm{ng} /$ $\mathrm{mL} \pm 1,5$ versus $1,7 \mathrm{ng} / \mathrm{mL} \pm 0,8 ; \mathrm{p}=0,91$ ) (Figura 2 ). Entre os pacientes com duração do DM $\leq 5$ anos, foi encontrada correlação inversa entre tempo de doença e o PC pós-glucagon, conforme indicado na Figura 3.

\section{FUNÇÃO PANCREÁTICA EM CRIANÇAS E ADULTOS COM DM 1}

Entre os pacientes com duração do $\mathrm{DM} \leq 5$ anos (22 pacientes), encontrou-se PC detectável basal e pós-estímulo em $35,3 \%$ e $64,7 \%$ dos casos (respectivamente) diagnosticados abaixo de 18 anos. Apenas cinco pacientes com curta duração do DM apresentavam idade de diagnóstico $\geq 5$ anos. Destes, quatro (80\%) tinham PC detectável, basal ou pós-estímulo. Nos pacientes com maior duração da doença, os três casos de PC detectável haviam sido diagnosticados abaixo de 18 anos. Em todos os três, a detecção ocorreu com ou sem estímulo.
$2 A$

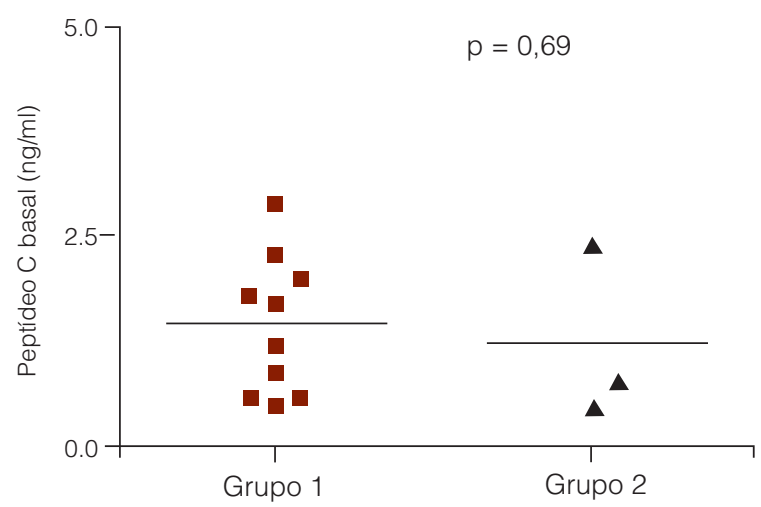

2B

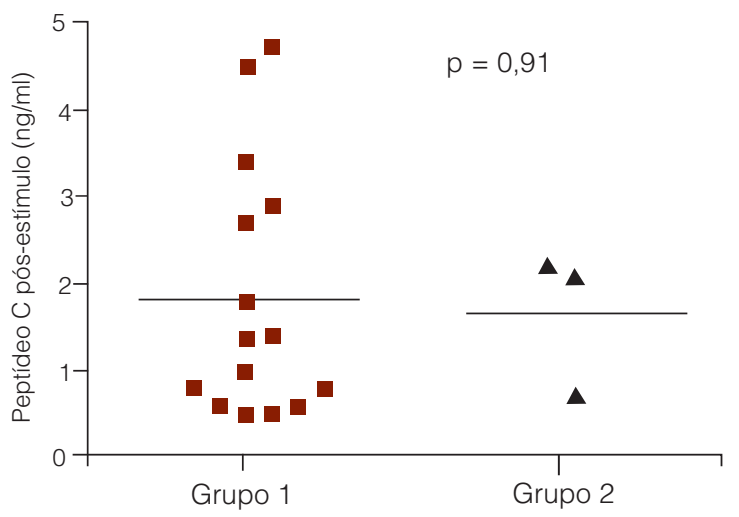

Figura 2. Títulos de peptídeo C (PC) detectável nos grupos 1 (diagnóstico de DM $1 \leq 5$ anos) e 2 (diagnóstico de DM $1>5$ anos). $2 A$. PC basal; 2B. PC pós-estímulo com glucagon EV. 
$3 A$

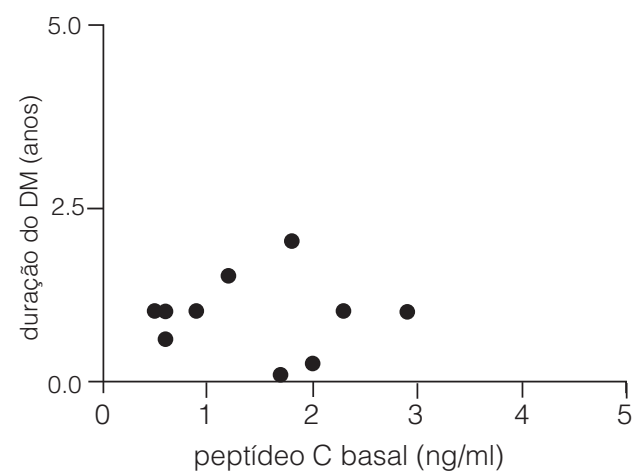

3B

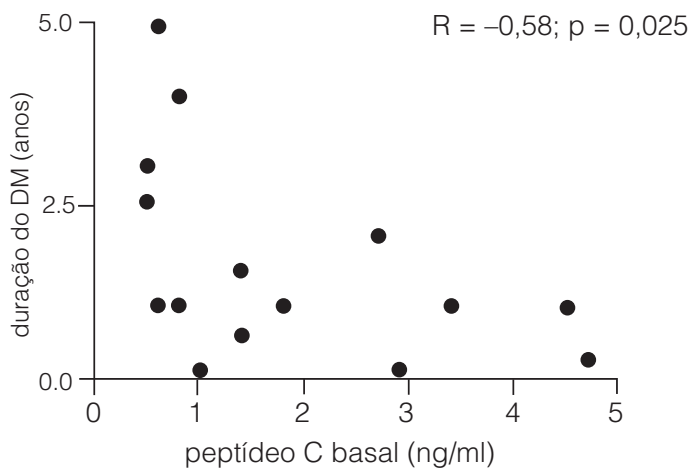

Figura 3. Correlação do peptídeo C e duração de doença no grupo 1 (diagnóstico $\leq 5$ anos). 3A: PC basal; 3B: PC pós-estímulo com glucagon EV.

\section{PC DETECTÁVEL EM PACIENTES COM DOENÇA DE LONGA DURAÇÃO}

Entre aqueles com diagnóstico há mais de cinco anos, foram encontrados três pacientes (em 2l) com PC detectável após estímulo $(0,8 ; 2,2$ e $2,4 \mathrm{ng} / \mathrm{mL})$. Todos apresentavam mais de dez anos de duração do DM (17, 10 e 37 anos), ausência de complicações graves da doença e anti-GAD negativo. Dois destes pacientes foram diagnosticados antes de dois anos de idade ( 1 e 1,5 anos) e apresentavam tireoidite de Hashimoto. Em um destes, havia ainda história de um irmão gêmeo univitelino com diagnóstico recente de DMl com anti-GAD (+) e tireoidite de Hashimoto. No caso restante, o diagnóstico foi feito aos 9 anos de idade e não havia outras doenças autoimunes concomitantes.

\section{DOSAGEM DO ANTI-GAD E ASSOCIAÇÃO COM PEPTÍDEO}

Foi realizada a dosagem do anti-GAD em 39 pacientes, sendo positiva em $46,2 \%$. O título médio nos pacientes com anti-GAD $(+)$ foi de $20,11 \pm 45,98 \mathrm{U} / \mathrm{mL}$. Não houve diferença na frequência de anti-GAD $(+)(60 \%$ versus $31,6 \%, \mathrm{p}=0,124)$ ou de seu título $(\mathrm{p}=0,743$; $11,52 \pm 12,68$ versus $37,27 \pm 79,41 \mathrm{U} / \mathrm{mL}$ ) entre os grupos 1 e 2, respectivamente. Não houve associação entre a presença de anti-GAD e do $\mathrm{PCl} / \mathrm{PC} 2$ detectá$\operatorname{vel}(\mathrm{p}=0,53$ e $\mathrm{p}=0,1$, respectivamente $)$ ou secreção de insulina preservada $(\mathrm{p}=0,667$ e $\mathrm{p}=0,702)$.

\section{DISCUSSÃO}

Este estudo avaliou a função das células $\beta$ pancreáticas de pacientes com DMl e tempo de doença variável por meio da dosagem do PC basal e após estímulo com glucagon. Embora a maior parte dos autores demonstre a superioridade do teste sob estímulo em relação ao basal, alguns estudos têm sugerido que a medida basal (em jejum ou randômica) pode ser suficiente (7). Na amostra do presente estudo, a utilização do glucagon como secretagogo da insulina foi crucial na identificação de parte considerável dos indivíduos com função residual pancreática.

Foi comparada a capacidade de secreção residual de insulina entre indivíduos com curta e longa duração de doença com nível semelhante de controle glicêmico. Conforme esperado pela própria história natural do $\mathrm{DMl}$, foi encontrada frequência mais elevada de PC detectável nos pacientes com duração do $\mathrm{DMl}$ de até cinco anos (8). No entanto, não houve diferença entre a presença de secreção preservada do PC nos dois grupos. É possível que isso se deva ao número reduzido de pacientes com esta característica na amostra ou, ainda, de início precoce da queda da função secretória, principalmente no primeiro ano de doença. Após cinco anos de doença, o PC estaria ainda detectável, porém não mais em nível compatível com a preservação da secreção. O fato de se ter encontrado correlação inversa entre $\mathrm{PC}$ e tempo de doença mesmo no grupo 1 corrobora esta hipótese.

Os dados da literatura são variáveis quanto à preservação da função pancreática ao diagnóstico e ao longo do curso da doença. No Diabetes Control and Complications Trial (DCCT), em indivíduos com diagnóstico $\leq 5$ anos, o PC pós-estímulo com refeição mista foi detectável (> 0,2 $\mathrm{nmol} / \mathrm{dL}$ ou $0,6 \mathrm{ng} / \mathrm{mL}$ ) em $33 \%$ dos pacientes com idade ao diagnóstico menor que 18 anos, e em $48 \%$ daqueles diagnosticados quando adultos (> 18 anos), estando preservada a função pancreática ao diagnóstico em 15\% (7-9). 
Em outro estudo mais recente, Steele e cols. (15) encontraram PC detectável em todos os pacientes com DMl recém-diagnosticado (diagnóstico inferior a 10 semanas), mas a resposta era de $52 \%$ da observada em indivíduos sem DM. Com estímulo suprafisiológico, déficit de secreção mais pronunciado foi evidenciado (com manutenção de cerca de $30 \%$ da resposta normal).

Neste estudo, observou-se $68 \%$ dos pacientes com cinco anos ou menos de doença com PC pós-estímulo detectável, portanto mais elevado do que no DCCT. Já a função se encontrava preservada em $27,3 \%$ dos pacientes, freqüência também superior ao descrito anteriormente $(9,10)$. A maior presença de indivíduos com secreção detectável neste estudo e em outros mais recentes $(5,15)$ pode ser em razão da utilização da insulinização intensiva de maneira mais precoce como tratamento padrão, logo após o diagnóstico de DMl, confirmando a importância do bom controle na manutenção da função residual das células $\beta$. É possível, também, que o diagnóstico de DMl esteja sendo feito de maneira cada vez mais precoce, pelo maior conhecimento da população de seus sinais e sintomas. Outro fator a ser considerado é o aumento do IMC e da resistência insulínica da população, que poderia contribuir para aumentar a secreção insulínica na fase pré-clínica do DMl. Entretanto, na nossa amostra, o sobrepeso e a obesidade não foram associados à preservação da função pancreática, talvez pelo número limitado de pacientes com excesso de peso. Outra possibilidade é a influência étnica, tendo em vista que a população em questão é miscigenada e isso poderia interferir na preservação da secreção insulínica. Pozzan e cols. (16) também encontraram proporção elevada de pacientes brasileiros com DMl com peptídeo C detectável nos dois primeiros anos de doença. Um número maior de indivíduos deve ser avaliado para a confirmação desses dados.

Não obstante a esperada queda pronunciada da produção de insulina em indivíduos com DMl de duração prolongada, dados anatomopatológicos recentes descreveram a presença de células contendo insulina neste grupo. Entretanto, o significado fisiológico deste achado ainda não é bem conhecido (5).

No DCCT, apenas $3 \%$ das crianças e $8 \%$ dos adultos apresentavam PC detectável após cinco anos de doença, porquanto a maioria tinha menos de dez anos do diagnóstico $(5,6)$. Na amostra do presente estudo, foi encontrada função pancreática residual em uma proporção mais elevada de pacientes com longa duração do DM (14,3\%, todos diagnosticados na infância). Uma frequência surpreendentemente alta (18\%) de indivíduos com DMl por mais de 50 anos e peptídeo $\mathrm{C}$ detectável também foi mostrada recentemente por Keenan e cols. (17).
Fatores capazes de influenciar a função das células $\beta$ no DMl também foram analisados. Embora Snoorgard e cols. (18) tenham encontrado associação entre a função residual pancreática e o sexo masculino, o presente estudo e outros autores não identificaram esta diferença entre os sexos. Também não foi encontrada qualquer associação entre etnia e capacidade de secreção de insulina. A divisão étnica entre brancos e não-brancos teve como base estudos prévios realizados pelo grupo, tendo em vista a população brasileira ser multiétnica e bastante miscigenada, o que impossibilita classificações fenotípicas $(13,14)$. Apesar das limitações desta classificação, visto que há considerável superposição entre os grupos, tem-se encontrado interessantes diferenças entre grupos de brancos e não-brancos com DMl, tanto do ponto de vista clínico quanto genético (19). Assim, acredita-se que, embora imperfeita, esta forma de nomenclatura seja a mais próxima do ideal na população estudada.

Nesta análise, a idade ao diagnóstico não se associou com a função pancreática residual, possivelmente em virtude da pequena quantidade de indivíduos diagnosticados abaixo de 5 ou acima de 18 anos de idade $(6,8)$. A influência da idade de diagnóstico de DMl na secreção de insulina também tem sido investigada por outros autores $(5,15)$. Karjalainen e cols. $(20)$ demonstraram que crianças apresentavam produção insulínica ao diagnóstico menor do que os adultos com $\mathrm{DMl}$ e outros estudos prospectivos confirmaram este achado. A perda progressiva da função pancreática ao longo dos meses parece ser discretamente maior em crianças do que em adultos $(5,21)$. Além disso, os adultos parecem partir de níveis iniciais de PC mais elevados, levando mais tempo (meses a anos) para atingir níveis indetectáveis, quando comparados às crianças (8).

O controle glicêmico também poderia ser capaz de influenciar a função pancreática no DMl e, conseqüentemente, os resultados deste estudo. Tanto a hiperglicemia aguda, pela glicotoxicidade nas células $\beta$, quanto a hipoglicemia pode interferir na secreção insulínica basal ou estimulada pelo glucagon, visto que esta última tem efeito inibitório mais expressivo (7). Portanto, apenas indivíduos com glicemia entre 70 e $200 \mathrm{mg} / \mathrm{dL}$ foram incluídos no estudo, conforme recomendação da The Immunology Diabetes Society (6). O controle glicêmico, a longo prazo, avaliado por meio da $\mathrm{HbAlc}$, também poderia influenciar na capacidade de secreção de insulina, embora isso ocorra em menor escala para o glucagon em relação aos demais estímulos utilizados (refeição mista, glicose, Sustacal ${ }^{\circledR}$ ) (7). Alguns trabalhos demonstraram níveis de PC detectáveis e preservados após refeição mista com freqüência significativamente maior em pacientes com diagnóstico recente 
de DMl e bom controle glicêmico $(5,10,15,22)$. Também foi observada correlação entre o nível de $\mathrm{HbAlc}$ e o PC pós-estímulo, o que sugere que melhor controle glicêmico favoreça a melhor função pancreática residual, e vice-versa, mesmo naqueles pacientes de longa duração de doença $(5,15)$. Os dois grupos (curta e longa duração de doença) apresentavam HbAlc semelhante, o que minimizaria o efeito do controle glicêmico na comparação entre ambos $(5,6,9)$.

Outro ponto estudado foi a ausência de associação entre a positividade para o anti-GAD e a função pancreática. Como descrito anteriormente $(12,23,24)$, foi encontrada baixa prevalência de anti-GAD nesta amostra, sem diferença na positividade do anti-GAD entre os grupos de curta $\mathrm{e}$ longa duração de doença, como seria esperado pela própria história natural do DMl $(2,11)$. Tal achado pode decorrer do tamanho da amostragem do presente estudo, pois verificou-se tendência a maior frequência de anti-GAD no grupo com diagnóstico há menos de cinco anos $(60 \%$ versus $31,6 \%)$. Pode-se especular que estas sejam características da população em questão, já que a maioria dos estudos prévios indicando prevalências mais elevadas foi realizada exclusivamente com caucasianos. Entretanto, Pardini e cols. (25) demonstraram previamente prevalência semelhante de anti-GAD nos pacientes com DMl do Brasil (mas com tempo de doença inferior a dois anos) e aquela obtida exclusivamente com caucasianos. Outra explicação para este achado seria a duração média do diabetes ter sido superior a um ano, mesmo no grupo de pacientes com diagnóstico recente. É possível que níveis de anti-GAD declinem de maneira significativa no primeiro ano de evolução da doença, até mesmo ao longo dos primeiros meses. Outra hipótese seria que alguns pacientes possam ter outras formas de diabetes, como DMlB, que não possui positividade para esse autoanticorpo, ou Maturity Onset Diabetes of the Young (MODY). São necessários estudos incluindo maior número de pacientes diagnosticados há menos de um ano para a confirmação desses dados.

Ainda não há papel definido dos anticorpos na progressão da lesão pancreática que leva ao DMl. Eles não parecem ter papel patogênico direto, mas sim serem liberados como reflexo da lesão celular (6). Apesar disso, estudos observacionais associaram a positividade para autoanticorpos com a perda progressiva da função das células $\beta$ no DMl. Wallesteen e cols. demonstraram que pacientes anticorpo antiilhota (ICA) (-) tinham peptídeo C pós-prandial mais elevados ao diagnóstico, e com 3, 9 e 12 meses do que aqueles ICA $(+)(26)$. Outros estudos prospectivos também associaram a presença de autoanticorpos, especificamente o anti-ICA $(+)$, à perda mais rápida da secreção insulínica, tanto em crianças $(5,27)$ quanto em adolescentes e adultos $(5,28)$. Contudo, alguns autores não encontraram qualquer relação entre a positividade para autoanticorpos e a perda da função pancreática (8). Nesta análise transversal, não foi observada associação entre a presença de anti-GAD ou do seu título com a dosagem do PC basal e pós-estímulo, tanto no total da amostra quanto em cada grupo isoladamente. Estudos longitudinais ainda são necessários para elucidar esta questão.

Em relação aos três pacientes com longa duração de DMl e secreção preservada de insulina, seria pertinente questionar se não teriam sido classificados erroneamente como DMl, não obstante a utilização dos critérios da ADA (12). Além de história clínica altamente sugestiva, nenhum tinha história familiar conhecida de diabetes em três gerações que sugerisse o diagnóstico de MODY, capaz de se apresentar clinicamente, algumas vezes, de maneira idêntica ao DMl. Também não havia outras características que apontassem formas mais raras de diabetes. O DM2 seria uma possibilidade remota, pela evolução clínica apresentada. Nenhum destes pacientes tinha antiGAD positivo, mas isso pode estar relacionado à longa duração da doença. Além disso, dois destes indivíduos apresentavam outra doença autoimune associada (tireoidite de Hashimoto) e um tinha um irmão gêmeo univitelino com anti-GAD (+). Estas características apontam o DMl como o diagnóstico mais provável.

Concluindo, proporção significativa dos pacientes com DMl apresentou alguma secreção residual de insulina, especialmente nos primeiros cinco anos da doença. Nos pacientes com curta duração do DMl, a correlação inversa entre tempo de doença e capacidade de secreção de insulina parece indicar queda contínua da função das células $\beta$ ao longo dos cinco primeiros anos após o diagnóstico. Alguns pacientes com longa duração do diabetes, mesmo maior do que dez anos, demonstraram ter ainda alguma produção de insulina. É possível que isso esteja associado a benefícios, como menor freqüência de hipoglicemia, melhor controle metabólico e menor risco de complicações microvasculares. São ainda necessários estudos longitudinais na população, com número maior de pacientes, para definir com mais clareza a história natural da função pancreática no DMl nestes indivíduos, com a finalidade de programar intervenções para a manutenção da secreção residual pancreática de insulina e atingir seus potenciais benefícios.

Agradecimento: A Novo Nordisk Farmacêutica do Brasil pela doação das ampolas de Glucagon ${ }^{\circledR}$.

Declaração: Os autores declaram não haver conflitos de interesses científico neste artigo. 


\section{REFERÊNCIAS}

1. Atkinson MA, Eisenbarth GS. Type 1 diabetes: new perspectives on disease pathogenesis and treatment. Lancet. 2001;358:221-9.

2. Daneman D. Type 1 Diabetes. Lancet. 2006;367:847-58.

3. Screenan S, Pick AJ, Levisettim, Baldwin AC, Pugh W, Polonsky KS. Increased beta-cell proliferation and reduced mass before diabetes onset in the nonobese diabetic mouse. Diabetes. 1999;48: 989-96.

4. Marchetti P, Dotta F, Zhiong L, Lupi R, Del Guerra S, Santangelo C, et al. The function of pancreatic islets isolated from type 1 diabetic patient. Diabetes Care. 2000;23:701-3.

5. Tsai EB, Sherry NA, Palmer JP, Herold KC, for the DPT-1 Study Group. The Rise and Fall of Insulin Secretion in type 1 diabetes mellitus. Diabetologia. 2006;49:261-70.

6. Palmer JP, Fleming GA, Greenbaum CJ, Herold KC, Jansa LD, Kolb H, et al. C-peptide is the appropriate outcome measure for type1 diabetes clinical trials to preserve beta cell function - ADA Workshop Report. Diabetes. 2004;53:250-64.

7. Vendrame F, Zappaterreno A, Dotta F. Markers of beta cell function in type 1 diabetes mellitus. Minerva Med. 2004;95:1-6.

8. Steffes MW, Sibley S, Jackson M, Thomas W. $\beta$-cell Function and the development of diabetes-related complications in diabetes control and complication trial. Diabetes Care. 2003;26:832-6.

9. Sherry NA, Tsai EB, Palmer JP, Herold KC. Natural history of beta cell function in type 1 diabetes. Diabetes. 2005;54:Suppl 2:S32-9.

10. The Diabetes Control and Complications Trial Research Group: the effect of intensive therapy on residual beta-cell function in patients with type 1 diabetes in the diabetes control and complications trial. Ann Intern Med. 1998;128:517-53.

11. Eisenbarth GS. Type 1 diabetes mellitus. In: Kahn CR, Weir GC, King GL, Moses AC, Smith RJ, Jacobson AM, editors. Joslin's diabetes mellitus. 14. ed. Boston, MA: Lippincott Williams \& Wilkins; 2005. p. 399-424.

12. American Diabetes Association (ADA). Diagnosis and classification of diabetes mellitus (position statements). Diabetes Care. 2005;28 Suppl 1:S37-42.

13. Rodacki M, Zajdenverg L, Tortora RP, Reis FA, Albernaz MS, Goncalves MR, et al. Characteristics of childhood and adult-onset type 1 diabetes in a multi-ethnic population. Diab Res Clin Pract. 2005;69:22-8.

14. Palatnik M, Silva Junior WA, Estalote AC, Oliveira JEP, Milech A, Zago MA. Ethnicity and type 2 diabetes in Rio de Janeiro, Brazil, with a review of the prevalence of the disease in Amerindians. Hum Biol. 2002;74:533-44

15. Steele C, Hagopian WA, Gitelman S, Masharani U, Cavaghan M, Rother $\mathrm{Kl}$, et al. Insulin secretion in type 1 diabetes. Diabetes. 2004;53:426-33.
16. Pozzan R, Dimetz T, Gazzola HM, Gomes MB. The C-peptide response to a standard mixed meal in a group of Brasilian type 1 diabetic patients. Braz J Med Biol Res. 1997;30(10):1169-74.

17. Keenan HA, Berger A, Sun JK, Eisenbath G, Doria A, King GL. Demonstration of islet cell function in patients with 50 years or longer of diabetes. Diabetes. 2007;56 Supl 1:A386.

18. Snorgaard O, Larsen LH, Binder C. Homogeneity in patter of decline of $\beta$-cell function in IDDM. Diabetes Care 15:1009-1015, 1992.

19. Rodacki M, Dantas J, Nabuco A, Barone B, et al. Diabetic ketoacidosis at the onset of type 1 diabetes:frequency and interfering factors. American Diabetes Association 67th Annnual Scentific Sessions. 2007;0918-P.

20. Karjalainen J, Salmela P, Ilonen J, Surcel HM, Knip M. A comparison of childhood and adult type 1 diabetes. N Engl J Med. 1989;320:881-6.

21. Leslie RDG, Williams R, Pozzilli P. Clinical Review: type 1 diabetes and latent autoimmune diabetes in adults: one end of the rainbow. J Clin Endocrinol Metab. 2006;91:1654-9.

22. Madsbad S, Krarup T, Regeur L, Faber OK, Binder C. Effect of strict blood glucose control on residual B-cell function in insulin-dependent diabetics. Diabetologia. 1981;20:530-4.

23. Rodacki M, Zajdenverg L, Albernaz MS, Bencke-Gonçalves MR, Milech A, Oliveira JE. Relationship between the prevalence of anti-glutamic acid descarboxilase autoantibodies and duration of type 1 diabetes mellitus in Brazilian patients. Braz J Med Biol Res. 2004;37(11):1645-50.

24. Dantas J, Almeida MH, Barone B, Suzundy R, Rodacki M, Zajdenverg $\mathrm{L}$, et al. Fatores clínico e epidemiológicos associados a positividade do anti-GAD em pacientes com diabetes mellitus tipo 1 (DM1) e longa duração de doença. Arq Bras Endocrinol Metab. 2007;51 Supl 1:S485.

25. Pardini VC, Mourao DM, Nascimento PD, Vivolo MA, Ferreira SR, Pardini $\mathrm{H}$. Frequency of islet cell autoantibodies (IA-2 and GAD) in young Brazilian type 1 diabetes patients. Braz J Med Biol Res. 1999;32:1195-8.

26. Wallensten $M$, Dahlquist $G$, Persson $B$, Landin-Olsson $M$, Lernmark $\AA$, Sundkvist G. Thalme B. Factors influencing the magnitude, duration, and rate of fall of b-cell function in type 1 (insulin dependent) diabetic children followed for two years from their clinical diagnosis. Diabetologia. 1988; 31:664-9.

27. Decochez K, Keymeulen B, Somers G. Use of an islet cell antibody assay to identify type 1 diabetic patients with rapid decrease in C-peptide levels after clinical onset: Belgium Diabetes Registry. Diabetes Care. 1996;19:1357-63.

28. Törn C, Olsson L, Lernmark A, Palmer JP, Arnqvist HJ, Blohmé G, et al. Prognostic fators for the course of $\beta$ cell function in autoimmune diabetes. J Clin Endocrinol Metab. 2000;85:4619-23. 